\title{
Regeln durch den Markt? Globale Kriminalitätsbekämpfung durch Lieferkettenregulierung
}

\author{
Anja P. Jakobi · Maike Stelter
}

Eingegangen: 4. Februar 2021 / Angenommen: 17. März 2021 / Online publiziert: 27. Mai 2021

(C) Der/die Autor(en) 2021

Zusammenfassung Globale Kriminalitätsbekämpfung umfasst zunehmend Aktivitäten nichtstaatlicher Akteure, um grenzüberschreitend Kriminalität zu verhindern. Der Beitrag analysiert die Regulierung von Lieferketten und die Einbindung von Unternehmen als Beispiel für den Ansatz, Marktregulierung zur Kriminalitätsbekämpfung zu nutzen. Dazu präsentiert er die Regulierung unterschiedlicher Sicherheitsrisiken am Anfang und Ende globaler Lieferketten, und zeigt inhärente Probleme von Lieferkettenregulierung als Instrument der Kriminalitätsbekämpfung auf, beispielsweise in Bezug auf Zielsetzung und Effektivität.

Schlüsselwörter Kriminalität · Regulierung $\cdot$ Lieferketten $\cdot$ Nichtstaatliche Akteure · Transnationale Governance

\section{The Market is the Master? Supply Chain Regulation as an Instrument of Global Crime Governance}

\begin{abstract}
Global crime governance is increasingly based on non-state actor activities in order to prevent cross-border crime. This article analyses the regulation of companies and global production chains as a proliferating instrument of global crime governance. For this purpose, it shows how this type of regulation addresses security risks at the beginning and the end of global production chains, and discusses inherent problems of this regulatory instrument, for instance ambiguous definitions of underlying problems or lacking effectiveness.
\end{abstract}

Prof. Dr. Anja P. Jakobi $(\bowtie) \cdot$ Maike Stelter Institut für Internationale Beziehungen, TU Braunschweig, Bienroder Weg 97, 38106 Braunschweig, Deutschland

E-Mail: a.jakobi@tu-braunschweig.de

Maike Stelter

E-Mail: m.stelter@tu-braunschweig.de 
Keywords Crime $\cdot$ Regulation $\cdot$ Supply chains $\cdot$ Non-state actors $\cdot$ Transnational governance

\section{Einleitung}

Kriminalitätsbekämpfung ist primär ein staatliches sicherheitspolitisches Interesse, doch viele Normen gegen Kriminalität wurden von nichtstaatlichen Akteuren ${ }^{1}$ geprägt (Andreas und Nadelmann 2006, S. 17-22; Jakobi und Haunschild 2016). Zivilgesellschaftliches Engagement ist heute regelmäßiger Bestandteil globaler Kriminalitätsbekämpfung, beispielsweise in der Korruptionsbekämpfung oder der Regulierung von Unternehmensaktivitäten in Kriegsökonomien (Enough Project 2012). Daneben kommt auch Unternehmen eine tragende Rolle in der Kriminalitätsbekämpfung zu, die von der globalen Implementierung von Standards und Corporate Social Responsibility bis zur Privatisierung von Sicherheit reicht (Liss und Sharman 2015; Flohr et al. 2010).

Die Regulierung von Lieferketten ist ein Instrument, das insbesondere auf die Kontrolle von und durch Unternehmen abzielt: Lieferketten bilden grenzüberschreitende Produktions- und Handelsprozesse ab, entweder innerhalb eines Unternehmens, Teilen eines Unternehmens, oder zwischen verschiedenen Unternehmen. Dabei können unterschiedliche Instrumente staatlicher oder nichtstaatlicher Akteure eingesetzt werden, um Firmen und Prozesse in der Lieferkette zu regulieren, zu beaufsichtigen und gegebenenfalls zu sanktionieren. Angewendet wird beispielsweise die verpflichtende Ausstellung von Herkunftsnachweisen, von Zertifikaten der nachhaltigen und legalen Produktion, Markierung und Nachverfolgung der Produkte, die Lizenzierung von Handelspartnern und Verkäufern, oder verpflichtende Risikoeinschätzungen.

Das Prinzip der Nachverfolgung wird auch im Bereich der Kriminalitätsbekämpfung zunehmend Bestandteil von Global Governance-Aktivitäten (Muirhead und Porter 2019). So wurden private Akteure zunächst in der Geldwäschebekämpfung dazu verpflichtet, Finanzströme stärker zu überprüfen, nachzuvollziehen und Risiken zu analysieren (Bergström et al. 2011). Der Kimberley-Prozess führte die Nachverfolgung von Diamanten ein, um die Finanzierung von Konfliktparteien zu verhindern (Haufler 2009). Mit dem Ziel der Kriminalitätsbekämpfung werden nichtstaatliche Akteure dabei systematisch in staatliche Aktivitäten eingebunden. Das Verhältnis von Staat und nichtstaatlichen Akteuren kann hierbei hierarchisch geprägt sein, indem der Staat private Akteure reguliert und in einem Prinzipal-Agent-Verhältnis mit dem direkten Auftrag der Kriminalitätsbekämpfung betraut - hier wären Banken und deren Transparenz- und Meldepflichten ein Beispiel. Zum anderen können Staat und nichtstaatliche Akteure analog eines Ressourcenaustauschs Informationen bündeln und gemeinsam staatliche und private Regulierung schaffen. Hier sind insbesondere zivilgesellschaftliche Akteure wichtig, aber auch Unternehmen und deren Wissen

\footnotetext{
${ }^{1}$ Im vorliegenden Artikel wird, abweichend vom ZfAS-Standard, bei personenbezogenen Substantiven die männliche grammatikalische Form verwendet. Die Autorinnen schließen damit Personen jeden Geschlechts gleichermaßen ein.
} 
über Lieferketten. Gerade Expertise kann in Märkten jedoch zwischen staatlichen und nichtstaatlichen Akteuren ungleich verteilt sein und so die Fähigkeit des Staates, Regeln zu setzen und hierarchisch zu steuern, unterlaufen. Die Zusammenarbeit zwischen Akteuren resultiert somit nicht notwendigerweise in einem Effektivitätsgewinn. Sie kann darüber hinaus auch das Ergebnis von Isomorphismus sein - der Tendenz, sozial akzeptierte und legitimierte Modelle der Kooperation zu implementieren, selbst wenn diese dysfunktional sind (Jakobi 2020, S. 96-109).

Im Folgenden zeigt der Beitrag die Nutzung von Instrumenten der Lieferkettenregulierung zur Kriminalitätsbekämpfung. Er präsentiert die Varianz der Lieferkettenregulierung, die mittlerweile sowohl zur Einschränkung bewaffneter Konflikte als auch zur Reduzierung von Umweltkriminalität oder zur Verhinderung von Gesundheitsschäden eingesetzt werden soll. In der abschließenden Zusammenfassung erfolgt eine Bewertung der Zunahme von Lieferkettenregulierung zur Kriminalitätsbekämpfung, insbesondere im Hinblick auf ihre Effektivität.

\section{Lieferkettenregulierung in der Kriminalitätsbekämpfung}

Die Kontrolle der Lieferketten von Rohstoffen wird insbesondere gegen die Finanzierung bewaffneter Konflikte eingesetzt. Dieses Risiko besteht in der Regel am Beginn einer globalen Lieferkette (Le Billon 2004). Das prominenteste Beispiel ist die Finanzierung von Bürgerkriegen durch Konfliktdiamanten und die Regulierung ihrer Lieferkette durch den Kimberley-Prozess. Vergleichbare Modelle der transnationalen Regulierung wurden jedoch auch in Bezug auf andere Rohstoffe eingeführt, die Konflikte finanzieren, insbesondere Konfliktmineralien wie Zinn, Coltan, Wolfram und Gold - als Gruppe bezeichnet als 3TG - sowie Holz.

Die Gruppe der 3TG sind insbesondere für die Elektronikindustrie eine zentrale Ressource und werden nur in wenigen Teilen der Welt abgebaut, insbesondere in der Demokratischen Republik Kongo und der umliegenden Region. Hier profitieren Rebellengruppen neben anderen illegalen Einnahmequellen direkt von den Erträgen der Minen, verbunden mit massiven Menschenrechtsverletzungen (Enough Project 2012; Radley und Vogel 2015). Die Verbindung zwischen den Konfliktmineralien und weltweiten Lieferketten wurde erstmals in den Berichten der Vereinten Nationen zwischen 2001 und 2003 hervorgehoben, indem Listen der beteiligten Unternehmen veröffentlicht wurden (UN 2002). Die Mineralien werden überwiegend in unreguliertem Kleinstbergbau abgebaut und lokal weitestgehend informell gehandelt (Taka 2016, S. 185). Der globale Markt der 3TG besteht aus einem breiten Spektrum an Unternehmen und zahlreichen Anwendungen der verschiedenen Rohstoffe in der Elektronikindustrie (Haufler 2015, S. 163). Unternehmen, die 3TG beziehen, wurden zunehmend verpflichtet ihre Lieferkette zu dokumentieren und Bürgerkriegsparteien als mögliche Profiteure auszuschließen. Dies erfolgte zunächst durch den US-amerikanischen Dodd-Frank Act, der Unternehmen betrifft, die an der US-Börse gelistet sind (US Securities and Exchange Commission 2010). Dazu gehört die Offenlegung der Bezugsquellen für Produkte, in denen die 3TG verwendet werden. Eine äquivalente Verordnung der Europäischen Union trat 2017 in Kraft (Europäisches Parlament und Europäischer Rat 2017). Um die Einfuhr von 3TG aus Konflikt- und 
Hochrisikogebieten zu vermeiden, verpflichtet diese Firmen, die die Überführung entsprechender Produkte in die EU anmelden, zur Etablierung von Sorgfaltspflichtsystemen, die auch Prüfungen durch unabhängige Dritte beinhalten können. Die EUVerordnung verweist hierfür explizit auf den Leitfaden der Organisation für wirtschaftliche Zusammenarbeit und Entwicklung (OECD) für Sorgfaltspflichten in Lieferketten für Minerale aus Konflikt- und Hochrisikogebieten (OECD 2019). Dieser beinhaltet Maßnahmen der Risikoprüfung und -vermeidung, inklusive dem Verweis auf Instrumente freiwilliger regionaler und privatwirtschaftlicher Initiativen.

Neben Mineralien können auch andere Rohstoffe zur Finanzierung und Aufrechterhaltung von Konflikten beitragen, insbesondere der globale Holzhandel (Price et al. 2007). Der Handel mit Holz und Holzprodukten kann hierbei sowohl im Rahmen illegaler Märkte, aber auch legaler Rodung zur Finanzierung von Konflikten beitragen. In bewaffneten Konflikten wird Holz zum Ankauf von Waffen verkauft, darüber hinaus sind der direkte Tausch gegen diese, die Nutzung privater Sicherheitskräfte als Milizen sowie diverse Zusammenhänge mit Formen der Finanzkriminalität und Korruption möglich (Blundell 2010). Holz wurde im Zusammenhang mit einzelnen Konflikten vom UN-Sicherheitsrat und der EU sanktioniert (UNSCR 2003, 2011; Europäischer Rat 2010). Neben diesen Sanktionen fällt der globale Handel einzelner seltener Baumarten unter das Washingtoner Artenschutzabkommen, jedoch bleibt die Lieferkette von Holz vorrangig Gegenstand europäischer oder nationaler Regulierung in den USA oder Australien, die Importeuren Sorgfaltspflichten auferlegt. So müssen Erstinverkehrbringer die Legalität der Produkte überprüfen und entsprechende Dokumentationen der Risikobewertung und ggf. -vermeidung anlegen. Bilaterale Partnerschaftsabkommen einzelner Länder mit der EU unterstützen dabei die Risikobewertung und -vermeidung, auch indem sie die Umsetzung von Regeln im Herkunftsland unterstützen. Die Zertifikate dieser Herkunftsländer erleichtern dann den Zugang zum europäischen Holzmarkt. Daneben kann eine selbstständige Risikoüberprüfung der Lieferketten durch die einführenden Unternehmen erfolgen, die eigene Kriterien oder Systeme von Drittanbietern nutzen. Diese Überprüfungen umfassen insbesondere die Markierung und Nachverfolgung der Produkte, regelmäßige Kontrollen bei Zulieferern, Überprüfungen anhand der Holz-DNA, oder die Nutzung kürzerer oder risikoärmerer Lieferketten und Produktionsstandorte. Private Zertifizierer, wie der Forest Stewardship Council, die ursprünglich die Nachhaltigkeit der Produkte ins Zentrum stellten, passten zudem in den vergangenen Jahren ihre Standards an die Erfordernisse für die legale Einfuhr an (Zeitlin und Overdevest 2020, S. 1). Die Regulierung der Lieferketten für Holz dient damit primär dem Ausschluss von Illegalität und damit verbundener Kriminalität (Europäisches Parlament und Europäischer Rat 2010). Konfliktholz als Bestandteil bewaffneter Konflikte - analog den Konfliktmineralien - wird zwar als Problem anerkannt, ist jedoch nicht Bestandteil der aktuellen Regulierungen, auch wenn dies durch zivilgesellschaftliche Organisationen gefordert wird (Global Witness 2017).

Lieferkettenregulierung erfolgt jedoch nicht nur aufgrund von Sicherheitsrisiken am Beginn der Lieferkette, wie beispielweise die Exportrestriktionen von Dual-UseGütern zeigen, bei denen die Risiken das Ende der Lieferkette betreffen (Weise et al. 2018). Neben diesem klassischen Sicherheitsrisiko rücken auch andere Lieferketten in den Fokus: So entstanden im pharmazeutischen Sektor in den letzten Jahrzehnten 
komplexe globale Lieferketten durch Verlagerung an Standorte mit geringen Lohnund Materialkosten und gleichzeitiger Spezialisierung auf bestimmte Herstellungsprozesse und Produkte. Insbesondere die Implementierung von Qualitätsstandards ist dabei schwierig, fehlende Implementierung kann jedoch zu Versorgungs- und Sicherheitsproblemen im Bereich Öffentliche Gesundheit führen. Probleme bereiten insbesondere Sub-Standard-Produkte: Ein dem Äußeren nach einwandfreiem Produkt kann unbemerkt falsche, schädliche oder nicht wirksame Substanzen enthalten. Während dieses Problem der Inverkehrbringung von Sub-Standard-Medikamenten durch kriminelle Akteure lange Zeit insbesondere Länder mit eingeschränkten Kontrollkapazitäten im medizinischen Sektor betraf (Zaman 2018), wurde die Komplexität der Lieferketten mittlerweile auch in Europa als Sicherheitsproblem der Gesundheitsvorsorge erkannt (OECD 2015, S. 81-83; Attaran et al. 2011, S. 330). Dabei werden Medikamente als ein spezifisches Markenpräparat präsentiert, dessen Inhaltsstoffe jedoch - anders als im Fall von Generika - nicht identisch mit diesem sind.

Die Corona-Pandemie machte die Relevanz von Lieferketten zur Versorgung mit medizinischen Gütern deutlich - so kam es, obwohl Deutschland Exporteur vieler medizinischer Güter ist, zu Beginn 2020 kurzfristig zu Versorgungsengpässen mit Schutzausrüstung durch die gestiegene Nachfrage auf dem Weltmarkt (Braml et al. 2020). Gleichzeitig führte die damit einhergehende Attraktivität des Marktes dazu, dass viele Produkte mit Qualitätsmängeln und gefälschten Zertifikaten in Verkehr gebracht wurden. So wurde oberflächlich die Versorgung gesichert, Produktsicherheit durch mangelhafte Zertifizierung jedoch vielfach erst später als Problem erkannt. Die aktuelle Nachfrage nach Corona-Impfstoff hat ebenfalls dazu geführt, dass in Deutschland und anderen Ländern erste Betrugsversuche mit angeblichem Impfstoff bekannt wurden (Polizeiliche Kriminalprävention der Länder und des Bundes 2021).

Grundsätzlich sind Fälschungen bei Produkten, deren Qualität ohne fachmännische Prüfung nicht einzuschätzen ist, zu erwarten, da das unbemerkte, systematische Unterlaufen von kostenintensiven Sicherheitsstandards attraktive Gewinne verspricht. In den Kontrollen der Gesundheitssysteme werden daher nicht nur Medikamente und ihre Zusammensetzung geprüft, sondern zudem die Lieferkette von der Produktion bis zur Abgabe - auch mithilfe der pharmazeutischen Industrie, die neben der Produktsicherheit das Interesse des Markenschutzes verfolgt. Verpackungen werden mit Sicherheitsmerkmalen wie RFID-Chips ${ }^{2}$ oder Hologrammen ausgestattet, die Größe von Tabletten standardisiert und die Handelskette nachvollziehbar gemacht (Muirhead und Porter 2019, S. 432-433). Der weltweite Handel mit Ausgangsstoffen für Medikamente - deren spezielle Erfordernisse sie teurer gegenüber vergleichbaren Ausgangstoffen für andere Anwendungen macht - erschwert Qualitätskontrollen, und macht den Markt prinzipiell attraktiv für kriminelle Gewinnabschöpfung. Gleichzeitig bestehen weitere Risiken entlang der Lieferkette: So werden die Medikamente selbst durch eine Vielzahl von Zwischenhändlern angeboten, was

\footnotetext{
2 RFID Chips sind sehr klein, preisgünstig und enthalten nur geringe Datenmengen, die mit einem Lesegerät ausgelesen werden. Sie finden sich heute in einer Vielzahl von Produkten, teilweise unter kleinen Aufklebern als Diebstahlschutz, in Reisepässen, oder sie werden auch als Chip für die Identifizierung von Tieren genutzt.
} 
die Möglichkeiten erhöht, selbst in stark regulierte Gesundheitssysteme Medikamentenfälschungen einzuschleusen (Powell 2010, S. 754-756). Die Reduktion der Anzahl von Zwischenhändlern, wie sie von manchen Firmen vorgenommen wird (OECD 2015, S. 101), schränkt diese Möglichkeiten ein, kann jedoch Prinzipien des im Gesundheitssystem zunehmend verbreiteten Marktwettbewerbs entgegenstehen.

Auch die Weltgesundheitsorganisation unterstreicht die Notwendigkeit der Nachverfolgung entlang der Lieferkette (WHO 2017, S. 56-58). Als relevanter internationaler Vertrag ist in diesem Bereich jedoch nur die Medicrime-Konvention des Europarates von 2011 (Europarat 2011) zu nennen, die versucht sowohl Herstellung und Handel von gefälschten Medikamenten zu kriminalisieren, ebenso die Fälschung von relevanten Dokumenten in der Lieferkette. Während sie damit primär ein Instrument des Strafrechts darstellt, berücksichtigt sie die grenzüberschreitende Lieferkette und ermöglicht vertiefte internationale Kooperation, nicht ausschließlich die Bestrafung im Hinblick auf das gefälschte Produkt (Boister 2018, S. 236-238; Romeo Casabona et al. 2017). In der Europäischen Union werden Lieferketten durch die Falsified Medicine Directive und damit verbundene Regulierung stärker überwacht: So müssen Verpackungen gekennzeichnet sein, Händler und Lieferanten für Ausgangsprodukte und Medikamente lizenziert sein und damit verbundene Produktions- und Qualitätsstandards erfüllen (Europäische Kommission 2019; Europäisches Parlament und Europäischer Rat 2011). Zusätzlich wurde seit 2016 ein System der Nachverfolgung implementiert, das Medikamente von ihrer Produktion bis zur Abgabe grenzüberschreitend überwacht (Europäische Kommission 2016). Ähnliche Systeme der Nachverfolgung durch Hersteller und Lieferanten werden auch in Ländern wie den USA eingeführt, um die Einschleusung von Fälschungen zu verhindern (Muirhead und Porter 2019, S. 433-434; Powell 2010, S. 757).

\section{Lieferkettenregulierung: Ausschluss oder Bekämpfung von Kriminalität?}

Die Regulierung von Lieferketten wird heute vielfach genutzt, um Kriminalitätsbekämpfung mithilfe privater Akteure grenzüberschreitend zu verwirklichen. Die zunehmende Akzeptanz entsprechender Instrumente als Mittel der Etablierung globaler Standards zeigt sich neben unseren Beispielen auch in Bezug auf andere Kriminalitätsformen, wie beispielsweise aktuelle Gesetzesvorhaben zu Lieferkettenregulierung gegen systematisches Unterlaufen von Arbeits- und Umweltschutzregeln (Schwab 2021; Crane et al. 2019). Die Regulierung von Lieferketten nutzt dabei primär die Größe der Märkte in der EU oder den USA, die trotz hoher Regulierungskosten für Lieferanten attraktiv sind.

Der Ansatz zeigt jedoch auch inhärente Probleme: Zunächst betrifft dies die Zielsetzung, die zwar oft auf Kriminalität rekurriert, aber üblicherweise mehrere Ziele in unklarer Priorisierung umfasst, die sich auch über die Zeit hinweg verändern können. So fokussierte die Regulierung von Holz erst angesichts des Scheiterns der Etablierung globaler Nachhaltigkeitsstandards für die Waldbewirtschaftung auf die Wichtigkeit von Legalität (Leipold et al. 2016). Das Problem der Konfliktfinan- 
zierung wird dabei anerkannt, die Auseinandersetzung mit der Problematik bleibt jedoch eingeschränkt. Ähnliche Probleme der Definition hat der Kimberley-Prozess, der unter Konfliktdiamanten nur Diamanten im Kontext von Bürgerkriegen fasst. Bei der Diskussion um gefälschte Medikamente ist hingegen auch in der Bekämpfung von illegalem Handel nicht immer eindeutig, ob der Markenschutz oder die Versorgungsicherheit Priorität haben.

Lieferkettenregulierung zielt auch primär darauf $a b$, eine bestimmte Lieferkette frei von unerwünschten Produkten und Prozessen zu halten, nimmt damit jedoch in Kauf, dass die Probleme verlagert, statt gelöst werden. Dies betrifft insbesondere Sicherheitsrisiken am Anfang der Lieferkette, bei denen sich die Anbieter neue Märkte suchen können, um Regulierungskosten und -implementierung zu umgehen. So zeigt der globale illegale Holzhandel trotz zunehmender Regulierung in westlichen Ländern anhaltend hohe Zahlen, was insbesondere durch eine Verschiebung der Lieferketten in andere Regionen erklärt wird (Gan et al. 2016, S. 51). Ob das Ziel von Lieferkettenregulierung also ist, bestimmte Formen von Kriminalität global zu bekämpfen, oder ob diese Kriminalität nur von bestimmten Märkten oder einzelnen Unternehmen ferngehalten werden soll, wird nicht immer deutlich. Damit verbunden ist auch das Risiko, dass über diese Verlagerung weniger kontrollierte Zwischenstationen der Lieferketten dazu genutzt werden, die ursprünglich ferngehaltenen Güter anschließend wieder in den attraktivsten Markt einzuschleusen.

Unternehmen haben angesichts komplexer globaler Lieferketten Probleme, die Standards zu garantieren: Untersuchungen der ersten drei Jahre des Dodd-Frank Acts zeigten, dass $80 \%$ der Unternehmen die Quelle ihrer Produkte nicht zweifelsfrei feststellen konnten und nur $1 \%$ mit hoher Sicherheit angeben konnte, konfliktfreie Produkte zu beziehen (Kim und Davies 2016). Unternehmen, die willentlich Standards unterlaufen oder sich für deren Abschaffung einsetzen, werfen zusätzlich die Frage auf, inwiefern betriebswirtschaftliche Ziele mit sicherheitspolitischen Interessen deckungsgleich sind. So sind Mitarbeiter von Banken zwar zur Bekämpfung von Terrorismusfinanzierung verpflichtet, es ist damit aber nicht garantiert, dass sie auch effektive Bekämpfung von Terrorismus leisten können. Auch die Versorgung mit medizinischen Gütern wird zunehmend als sicherheitsrelevant anerkannt, aber die Komplexität dieser Lieferketten steht in einem gewissen Widerspruch zur Versorgungsicherheit. Dieses Versorgungproblem ist dabei nicht neu (Bundesinstitut für Arzneimittel und Medizinprodukte 2021), sondern wird nur in der Pandemie besonders deutlich. So zeigt dieser Fall auch, wie die Komplexität von Lieferketten selbst zunehmend zu einem Risiko wird. Die Regulierung von Lieferketten zielt jedoch primär darauf $\mathrm{ab}$, diese Komplexität abzubilden und mit zunehmender Dokumentationspflicht zu verbinden, was nicht notwendigerweise die qualitative und quantitative Versorgungsicherheit verbessert.

Lieferkettenregulierung verfolgt mit Kriminalitätsbekämpfung zwar ein wichtiges Ziel, die Komplexität der regulierten Lieferketten macht eine effektive Kontrolle allerdings kaum möglich. Im Gegenteil: Zu der Komplexität der Lieferketten tritt dann eine Komplexität der Dokumentation hinzu, die nicht notwendigerweise vor kriminellem Handeln schützt, sondern auch neue Möglichkeiten schafft, bestehende Regeln zu unterlaufen. Darüber hinaus können einige der Probleme, die Lieferkettenregulierung versucht zu vermeiden, durch die Tatsache verstärkt werden, dass diese 
Regulierung mehr darauf abzielt, Kriminalität fernzuhalten, als sie durch weniger komplexe Lieferketten, andere Formen der Produktion oder durch verbindliche und implementierte internationale Standards zu bekämpfen: So sind gerade Produkte, Händler oder Hersteller, die sich nur scheinbar an die Regeln der Lieferkettenregulierung halten, durch die dadurch reduzierten Kosten im Markt besonders kompetitiv. Zusätzlich muss die Frage gestellt werden - und zwar sowohl bei Risiken am Beginn als auch am Ende der Lieferketten -, wer letztendlich vor Kriminalität geschützt werden soll, und ob dies durch diese Regulierungsform langfristig gelingen kann. Gerade da der relative Anteil der USA oder EU an den Weltmärkten abnimmt, scheint diese Form der Kriminalitätsbekämpfung ungeeignet, einer Verlagerung von durch Kriminalität profitierender Lieferketten in andere, attraktive Märkte der Welt zu verhindern. Insofern kann Lieferkettenregulierung weiterhin nur ein Instrument unter vielen sein, um Kriminalität - innerhalb und außerhalb von Landesgrenzen zu begegnen.

Funding Open Access funding enabled and organized by Projekt DEAL.

Open Access Dieser Artikel wird unter der Creative Commons Namensnennung 4.0 International Lizenz veröffentlicht, welche die Nutzung, Vervielfältigung, Bearbeitung, Verbreitung und Wiedergabe in jeglichem Medium und Format erlaubt, sofern Sie den/die ursprünglichen Autor(en) und die Quelle ordnungsgemäß nennen, einen Link zur Creative Commons Lizenz beifügen und angeben, ob Änderungen vorgenommen wurden.

Die in diesem Artikel enthaltenen Bilder und sonstiges Drittmaterial unterliegen ebenfalls der genannten Creative Commons Lizenz, sofern sich aus der Abbildungslegende nichts anderes ergibt. Sofern das betreffende Material nicht unter der genannten Creative Commons Lizenz steht und die betreffende Handlung nicht nach gesetzlichen Vorschriften erlaubt ist, ist für die oben aufgeführten Weiterverwendungen des Materials die Einwilligung des jeweiligen Rechteinhabers einzuholen.

Weitere Details zur Lizenz entnehmen Sie bitte der Lizenzinformation auf http://creativecommons.org/ licenses/by/4.0/deed.de.

\section{Literatur}

Andreas, P., \& Nadelmann, E. (2006). Policing the globe. Criminalization and crime control in international relations. Oxford: Oxford University Press.

Attaran, A., Bate, R., \& Kendall, M. (2011). Why and how to make an international crime of medicine counterfeiting. Journal of International Criminal Justice, 9(2), 325-354.

Bergström, M., Svedberg Helgesson, K., \& Mörth, U. (2011). A new role for for-profit actors? The case of anti-money laundering and risk management. Journal of Common Market Studies, 49(5), 1-22.

Blundell, A. G. (2010). Forests and conflict: The financial flows that fuel war. Washington DC: Program on Forest.

Boister, N. (2018). An introduction to transnational criminal law. Oxford: Oxford University Press.

Braml, M., Teti, F., \& Aichele, R. (2020). Apotheke der Welt oder am Tropf der Weltwirtschaft? Deutschlands Außenhandel auf dem Markt für Arzneien und medizinische Ausrüstungen. ifo Schnelldienst, 73(5), 35-42.

Bundesinstitut für Arzneimittel und Medizinprodukte (2021). Lieferengpässe für Humanarzneimittel. https://www.bfarm.de/DE/Arzneimittel/Arzneimittelzulassung/Arzneimittelinformationen/Liefereng paesse/_functions/Filtersuche_Formular.html?queryResultId=null\&pageNo=0. Zugegriffen: 25. Jan. 2021.

Casabona, R. C. M., Urruela, M. A., Jiménez, P. N., \& Alarcón-Jiménez, Ó. (2017). International strategies in fighting against medicaments fraud and other similar offences. The Medicrime Convention. Crime, Law and Social Change, 68(1-2), 95-122. 
Crane, A., LeBaron, G., Allain, J., \& Behbahani, L. (2019). Governance gaps in eradicating forced labor. From global to domestic supply chains. Regulation and Governance, 13(1), 86-106.

Enough Project (2012). From congress to Congo. Turning the tide on conflict minerals, closing loopholes, and empowering miners. https://enoughproject.org/files/ConflictMinerals_CongoFINAL.pdf. Zugegriffen: 26. Jan. 2021.

Europäische Kommission (2016). Commission delegated regulation (EU) 2016/161 of 2 October 2015 supplementing Directive 2001/83/EC of the European Parliament and of the Council by laying down detailed rules for the safety features appearing on the packaging of medicinal products for human use. https://ec.europa.eu/health/sites/health/files/files/eudralex/vol-1/reg_2016_161/reg_2016_161_ en.pdf. Zugegriffen: 26. Jan. 2021.

Europäische Kommission (2019). Falsified medicines. https://ec.europa.eu/health/human-use/falsified_ medicines_de. Zugegriffen: 26. Jan. 2021.

Europäischer Rat (2010). Council Decision 2010/788/CFSP of 20 December 2010 concerning restrictive measures against the Democratic Republic of the Congo and repealing Common Position 2008/369/CFSP. http://eur-lex.europa.eu/LexUriServ/LexUriServ.do?uri=OJ:L:2010:336:0030: 0042:EN:PDF. Zugegriffen: 26. Jan. 2021.

Europäisches Parlament \& Europäischer Rat (2010). Regulation (EU) Nr. 995/2010 of the European Parliament and of the Council of 20 October 2010 laying down the obligations of operators who place timber and timber products on the market. https://eur-lex.europa.eu/LexUriServ/LexUriServ.do?uri=OJ: L:2010:295:0023:0034:EN:PDF. Zugegriffen: 27. Jan. 2021.

Europäisches Parlament \& Europäischer Rat (2011). Directive 2011/62/EU of the European Parliament and of the Council of 8 June 2011. Amending Directive 2001/83/EC on the Community code relating to medicinal products for human use, as regards the prevention of the entry into the legal supply chain of falsified medicinal product. https:/ec.europa.eu/health/sites/health/files/files/eudralex/vol1/dir_2011_62/dir_2011_62_en.pdf. Zugegriffen: 26. Jan. 2021.

Europäisches Parlament \& Europäischer Rat (2017). Regulation (EU) 2017/821 of the European Parliament and of the Council of 17 May 2017 laying down supply chain due diligence obligations for Union importers of tin, tantalum and tungsten, their ores, and gold originating from conflictaffected and high-risk areas. https://eur-lex.europa.eu/legal-content/EN/TXT/PDF/?uri=CELEX: 32017R0821\&from=EN. Zugegriffen: 27. Jan. 2021.

Europarat (2011). Council of Europe Convention on the counterfeiting of medical products and similar crimes involving threats to public health. https://rm.coe.int/CoERMPublicCommonSearchServices/ DisplayDCTMContent?documentId=090000168008482f. Zugegriffen: 26. Jan. 2021.

Flohr, A., Ried, L., Schwindenhammer, S., \& Wolf, K. D. (2010). The role of business in global governance. Corporations as norm entrepreneurs. Basingstoke: Macmillan.

Gan, J., Cerutti, P., Masiero, M., Pettenella, D., Andrighetto, N., \& Dawson, T. (2016). Quantifying illegal logging and related timber trade. In D. Kleinschmit, S. Mansourian, C. Wildburger \& A. Purret (Hrsg.), Illegal logging and related timber trade. Dimensions, drivers, impacts and responses (S. 37-60). Vienna: IUFRO.

Global Witness (2017). The EU's responsibility to act on conflict timber. A new framework for action. https://www.globalwitness.org/documents/19084/Conflict_Timber_Briefing_June_2017_Zvklwk4. pdf. Zugegriffen: 26. Jan. 2021.

Haufler, V. (2009). The Kimberley process certification scheme: an innovation in global governance and conflict prevention. Journal of Business Ethics, 89(4), 403-416.

Haufler, V. (2015). Corporations, conflict minerals, and corporate social responsibility. In A. Lim \& K. Tsutsui (Hrsg.), Corporate social responsibility in a globalizing world (S. 149-180). Cambridge: Cambridge University Press.

Jakobi, A.P. (2020). Crime, security and global politics. An introduction to global crime governance. London: Macmillan, Red Globe Press.

Jakobi, A.P., \& Haunschild, J. (2016). Global Crime Governance: Perspektiven und Grenzen transnationaler Kooperation. Zeitschrift für Außen- und Sicherheitspolitik, 9(1), 1-11.

Kim, Y.H., \& Davis, G. F. (2016). Challenges for global supply chain sustainability. Evidence from conflict minerals reports. Academy of Management Journal, 59(6), 1896-1916.

Le Billon, P. (2004). The geopolitical economy of 'resource wars'. Geopolitics, 9(1), 1-28.

Leipold, S., Sotirov, M., Frei, T., \& Winkel, G. (2016). Protecting 'first world' markets and 'third world' nature. The politics of illegal logging in Australia, the European Union and the United States. Global Environmental Change, 39(4), 294-304.

Liss, C., \& Sharman, J. C. (2015). Global corporate crime-fighters. Private transnational responses to piracy and money laundering. Review of International Political Economy, 22(4), 693-718. 
Muirhead, J., \& Porter, T. (2019). Traceability in global governance. Global Networks, 19(3), 423-443.

OECD - Organization for Economic Co-operation and Development (2015). Illicit trade. Converging criminal networks. https://www.oecd-ilibrary.org/docserver/9789264251847-en.pdf?expires $=16159747$ 54\&id=id\&accname=ocid41021573\&checksum=F03F17BC958D40CC0E5242985CD6951E. Zugegriffen: 16. März 2021.

OECD (2019). OECD-Leitfaden für die Erfüllung der Sorgfaltspflicht zur Förderung verantwortungsvoller Lieferketten für Minerale aus Konflikt- und Hochrisikogebieten. Dritte Ausgabe. https:// www.oecd-ilibrary.org/docserver/3d21faa0-de.pdf?expires $=1621337460 \&$ id=id\&accname=guest\& checksum=7683A2D81F0358E2F45ED34A256E7E01. Zugegriffen: 18. Mai 2021.

Polizeiliche Kriminalprävention der Länder und des Bundes (2021). Vorsicht vor Betrug mit CoronaImpfstoff. https://www.polizei-beratung.de/startseite-und-aktionen/aktuelles/detailansicht/vorsichtvor-betrug-mit-corona-impfstoff/. Zugegriffen: 27. Jan. 2021.

Powell, A. (2010). Benchmark legislation. A measured approach in the fight against counterfeit pharmaceuticals. Hastings Law Journal, 61(3), 749-772.

Price, S., Donovan, D., \& de Jong, W. (2007). Confronting conflict timber. In W. de Jong, D. Donovan \& K. I. Abe (Hrsg.), Extreme conflict and tropical forests (S. 117-132). Dordrecht: Springer.

Radley, B., \& Vogel, C. (2015). Fighting windmills in Eastern Congo? The ambiguous impact of the 'conflict minerals' movement. Extractive Industries and Society, 2(3), 406-410.

Schwab, T. (2021, 14. Jan.). Streit über Lieferkettengesetz. Menschenrechte? Das hat Zeit. Frankfurter Rundschau. https://www.fr.de/wirtschaft/lieferkettengesetz-streit-bundestag-menschenrechte-dashat-zeit-90167616.html. Zugegriffen: 25. Jan. 2021.

Taka, M. (2016). A critical analysis of human rights due diligence frameworks for conflict minerals. Challenges for the electronics industries. Cambridge University Press. https://www.cambridge.org/core/ services/aop-cambridge-core/content/view/127F34782BE10628E525F1679F00667A/97817806874 21c8_p183-208_CBO.pdf/critical_analysis_of_human_rights_due_diligence_frameworks_for_confl ict_minerals_challenges_for_the_electronics_industries.pdf. Zugegriffen: 21. Jan. 2021.

U.S. Securities and Exchange Commission (2010). Dodd-Frank Wall Street reform and consumer protection act, pub. L. No. 1376. https://www.congress.gov/111/plaws/publ203/PLAW-111publ203.pdf. Zugegriffen: 30. Jan. 2021.

UN - United Nations (2002). Final report of the panel of experts on the illegal exploitation of natural resources and other forms of wealth of the Democratic Republic of the Congo, UN Doc. S/2002/1146. https://undocs.org/S/2002/1146. Zugegriffen: 21. Jan. 2021.

UNSCR - United Nations Security Council (2003). Resolution 1478. The situation in Liberia, 6 May 2003. S/RES/1478 (2003). http://unscr.com/en/resolutions/doc/1478. Zugegriffen: 30. Jan. 2021.

UNSCR (2011). Resolution 2021. The situation concerning the Democratic Republic of the Congo, 29 November 2011. S/RES/2021. http://unscr.com/en/resolutions/doc/2021. Zugegriffen: 30. Jan. 2021.

Weise, R. A., Hund, G., \& Carr, G. (2018). Export controls and counterproliferation finance. Two sides of the same underlying illegal WMD activity. The Nonproliferation Review, 25(1-2), 129-145.

World Health Organization (2017). WHO global surveillance and monitoring system for substandard and falsified medical products. Reports and executive summary. https://apps.who.int/iris/bitstream/ handle/10665/326708/9789241513425-eng.pdf. Zugegriffen: 17. März 2021.

Zaman, M.H. (2018). Bitter pills. The global war on counterfeit drugs. Oxford: Oxford University Press.

Zeitlin, J., \& Overdevest, C. (2020). Experimentalist interactions. Joining up the transnational timber legality regime. https://onlinelibrary.wiley.com/doi/epdf/10.1111/rego.12350. Zugegriffen: 26. Jan. 2021. 